University of Montana

ScholarWorks at University of Montana

Chemistry and Biochemistry Faculty

Publications

Chemistry and Biochemistry

$11-15-2001$

\title{
Micromagnetics Simulation of Deep-Submicron Supermalloy Disks
}

\author{
Scott L. Whittenburg \\ University of Montana - Missoula, scott.whittenburg@umontana.edu \\ NgocNga Dao \\ University of New Orleans \\ R. P. Cowburn \\ University of Durham
}

Follow this and additional works at: https://scholarworks.umt.edu/chem_pubs

Part of the Chemistry Commons, and the Physics Commons

Let us know how access to this document benefits you.

\section{Recommended Citation}

Whittenburg, Scott L.; Dao, NgocNga; and Cowburn, R. P., "Micromagnetics Simulation of Deep-Submicron Supermalloy Disks" (2001). Chemistry and Biochemistry Faculty Publications. 42.

https://scholarworks.umt.edu/chem_pubs/42

This Article is brought to you for free and open access by the Chemistry and Biochemistry at ScholarWorks at University of Montana. It has been accepted for inclusion in Chemistry and Biochemistry Faculty Publications by an authorized administrator of ScholarWorks at University of Montana. For more information, please contact

scholarworks@mso.umt.edu. 


\title{
Micromagnetics simulation of deep-submicron supermalloy disks
}

\author{
N. Dao and S. L. Whittenburga) \\ Advanced Materials Research Institute and Department of Chemistry, University of New Orleans, \\ New Orleans, Louisiana 70148 \\ R. P. Cowburn \\ Department of Physics, University of Durham, Durham DH1 3LE, United Kingdom
}

(Received 30 January 2001; accepted for publication 25 August 2001)

\begin{abstract}
The results of recent micromagnetic simulations of deep submicron supermalloy disks are presented. A recent experimental measurement of the hysteresis and magnetic domain structure in supermalloy disks with diameters ranging from 55 to $500 \mathrm{~nm}$ and thickness ranging from 6 to $15 \mathrm{~nm}$ has been reported. Our micromagnetic simulations show remarkable agreement with the experimental hysteresis loops. The simulation results show that for thin or small diameter disks a single magnetic domain exists with all spins aligned. The hysteresis loop represents free rotation of these spins. For larger diameter disks or as the thickness increases the hysteresis loops change shape due to the appearance of a single vortex state appearing at low applied fields. (C) 2001 American Institute of Physics. [DOI: 10.1063/1.1412838]
\end{abstract}

\section{INTRODUCTION}

Dot arrays of magnetic material have been studied both experimentally and theoretically in recent years as media manufactured using dot arrays may find a myriad of technological applications such as magnetic sensors and ultrahigh density storage media. There has been significant scientific interest in the effect of size and shape of dot arrays on magnetic characteristics. ${ }^{1-3}$ In particular, the circular shape has received a lot of attention. ${ }^{2,4-6}$ In those studies, ${ }^{4-6}$ magnetic properties such as the magnetic domain structure as a function of thickness as well as diameter of the dots were examined.

Understanding the formation and annihilation of magnetic domains is important since domains consisting of vortex structures can destroy the information stored in magnetic memory. Also by allowing study of the magnetic domain structure through each step in a hysteresis loop, micromagnetic simulations may provide a deeper understanding of the microscopic changes in the magnetization of the material. In this article, we present the results of simulations on supermalloy dots and examine the influence of the particle's diameter on nucleation and annihilation fields along with the magnetic domain structure.

\section{PROCEDURE}

The object oriented microMagnetic framework $(\mathrm{OOMMF})^{7}$ obtained from The National Institute of Standards and Technology is an excellent tool for simulation of micromagnetic problems. ${ }^{8}$ The exchange energy is computed by an eight-neighbor dot product. ${ }^{9}$ The constant volume charge method ${ }^{10}$ is used for the magnetostatic energy in which spins are on the corners of grids and with approximation of the magnetic volume charge $\rho=-\operatorname{div} \mathbf{m}$. The mag-

a) Author to whom correspondence should be addressed; electronic mail: swhitten@uno.edu netization of the sample is followed as a function of time using the Laudau-Lifshitz-Gilbert equation of motion on a two-dimensional (2D) grid with 3D magnetization spins.

Experimental work on supermalloy $\left(\mathrm{Ni}_{80} \mathrm{Fe}_{14} \mathrm{Mo}_{5}\right)$ circular dots has recently been reported. ${ }^{5}$ In these experiments, supermalloy dots ranging in thickness from 6 to $15 \mathrm{~nm}$ and diameters ranging from 55 to $500 \mathrm{~nm}$ were fabricated and studied using a high sensitivity magneto-optical method. Our simulation used a $2 \times 2$ array of dots for each diameter. The size and spacing of the dots was chosen to reproduce the experimental dots. For larger dots, the spacing between the dots was equal to or greater than their diameter. However, for dots with diameters below $150 \mathrm{~nm}$ the spacing was two or three times greater than their diameter. With this arrangement, the dots should be noninteracting. However, the possible magnetostatic interaction between the dots is included in the calculation.

In these simulations, we used a saturation magnetization of $8.0 \times 10^{5} \mathrm{~A} / \mathrm{m}$ and an exchange stiffness constant of 1.05 $\times 10^{-11} \mathrm{~J} / \mathrm{m}$. An anisotropy constant $K_{1}$ of $3 \mathrm{~J} / \mathrm{m}^{3}$ was taken as an approximation based on molybdenum permalloy ${ }^{11}$ with in-plane anisotropy. This value of $K_{1}$ is close to $0 \mathrm{~J} / \mathrm{m}^{3}$, the OOMMF default value for permalloy. It is unlikely that such small differences in the value for the anisotropy constant can be observed in the simulations, however, the value of $3 \mathrm{~J} / \mathrm{m}^{3}$ was used to conform to the experimentally obtained value. The default of damping constant of 0.5 was used. The sample was discretized into a cell size of $10 \mathrm{~nm}$. As a test, a $300 \mathrm{~nm}$ disk was also simulated with a cell size of $5 \mathrm{~nm}$ and gave similar results. The roughness of the circular shape is probably unimportant since the hysteresis loops are the same irrespective of the in-plane magnetization direction.

All of our results were performed on a $300 \mathrm{MHz}$ DEC Alpha workstation. The computation time for the largest dot was approximately 1 day, and for the smallest one a few hours. For all of the dots, there were 100 steps taken for each 


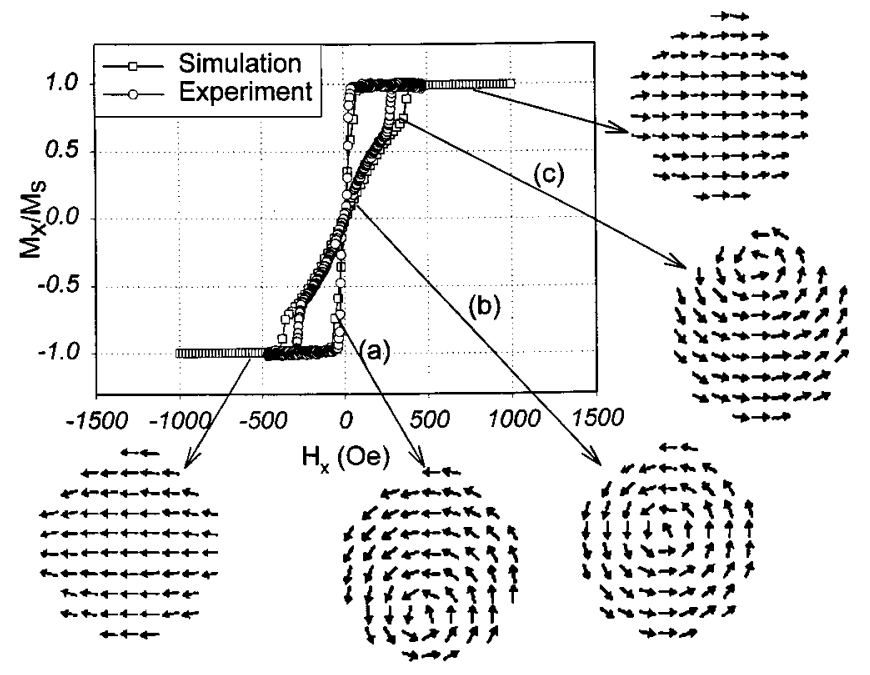

FIG. 1. The simulated data (square) and the experimental data (circle) for diameter of $300 \mathrm{~nm}$ and thickness of $10 \mathrm{~nm}$ : (a) nucleation of a vortex, (b) vortex structure, and (c) the annihilation state. Every third spin is shown.

forward and reverse loop in the hysteresis loop with a maximum external applied field of $100 \mathrm{mT}$.

\section{RESULTS}

In general, our simulation results agree well with the experimental hysteresis loops. There are two basic types of hysteresis loops for these samples. One type shows a simple loop corresponding to Stoner-Wohlfarth-like free rotation of the spins. The other type displays a more complex hysteresis loop. When the sample is thick or the diameter is large, the demagnetizing field can overcome the exchange field, and curl states may appear, producing an hysteresis loop with kinks. However, in the very thin or small diameter samples, because of the nature of dimensional confinement, the exchange field is the dominant contribution. This gives rise to single domain structures and a free-rotation hysteresis loop. The shapes of the both type of loop are the same as those observed experimentally. A measure of agreement for those loops with curl or vortex states is the value of the nucleation field, the value of the field at which the vortex first appears. For all dot diameters the nucleation field obtained from the simulation agrees with the experimental value to within 20 Oe. At the largest diameter, $500 \mathrm{~nm}$, the simulated hysteresis loop is exactly the same as the experimental loop; the nucleation field agrees within 2 Oe.

The magnetic domain structures were mapped as a function of applied field to observe how magnetic domains change during the hysteresis loop. Samples with a diameter of $300 \mathrm{~nm}$ display a complex hysteresis loop as shown in Fig. 1. The simulated hysteresis loop agrees very well with the experimental hysteresis loop. At the saturation field, all magnetization spins are in the direction of the applied field. The dramatic increase in the magnetization as a function of applied field at point (a) is due to the creation or nucleation of the vortex. At point (a) a vortex or curl state appears. The lowest magnetization corresponds to a vortex state centered in the sample [point (b)]. The diagonal line in the hysteresis loop is the motion of vortex across the sample. This move-

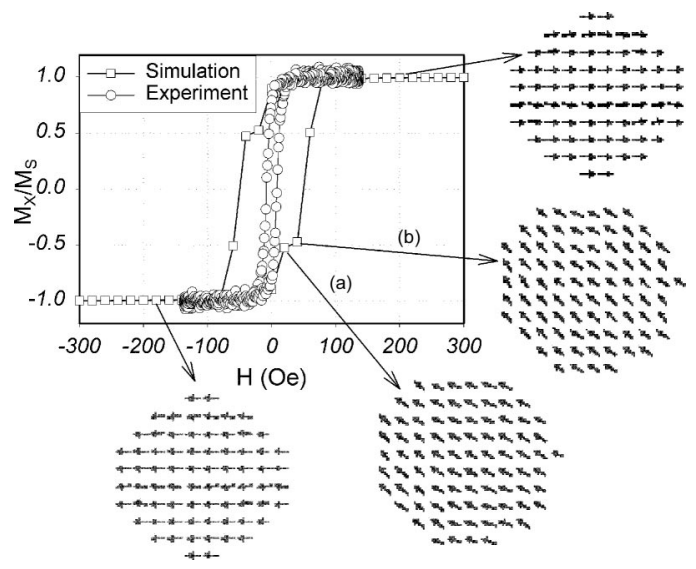

FIG. 2. The simulated hysteresis loop (square) and the experimental hysteresis loop (circle) for diameter of $100 \mathrm{~nm}$ and thickness of $10 \mathrm{~nm}$ : (a) all of magnetization spins aligned in one direction, and (b) at the kink point in this loop.

ment of the vortex across the dot continues until there is a sudden increase in magnetization due to the annihilation of the vortex [point (c)].

As an example of the samples with hysteresis loops displaying coherent rotation the loop for a dot with a diameter of $100 \mathrm{~nm}$ is given in Fig. 2. The simulated hysteresis loop and experimental loop show the same general shape; however, the simulated coercive field is about six times higher than the experimental coercive field. There is a kink in both simulated hysteresis loops. Point (a) corresponds to a domain pattern where the majority of spins are aligned in the same direction. At the kink (b) some of the spins become slightly more tilted relative to the applied field. This state is similar to the " $S$ " state observed in other micromagnetic studies.

All of the simulated hysteresis loops have the same general shape as the experimental hysteresis loops except for samples with a diameter of $150 \mathrm{~nm}$. For this sample, the simulated hysteresis loop has a higher coercivity than the experimental curve. Dots with a diameter of $150 \mathrm{~nm}$ are the borderline between supporting the vortex domain and the single domain.

The various contributions to the energy of each dot are important in understanding the reversal mechanism. To sustain a vortex structure the demagnetizing energy decreases at the expense of the exchange energy. In a vortex the spins at the edge of the dot are aligned relative to the edge. As the vortex is annihilated, the exchange energy drops rapidly as the spins become aligned (Fig. 3).

By combining the simulation results with those obtained from the experimentally measured hysteresis loops a magnetic "phase" diagram may be obtained. Such a diagram is shown in Fig. 4. Our results suggest that the diameter and thickness of the dots determines whether the magnetic domain structure will be single domain (SD), a vortex state (VS), or multidomain (MD). The characteristic length scale in these systems is the exchange length, $l_{\mathrm{ex}}=\left(2 \mathrm{~A} / \mu_{o} M_{s}\right)^{1 / 2}$, because a dominant exchange interaction will lead to alignment of the spins and result in a single domain state. Thus, a phase diagram may be obtained in terms of two dimensionless parameters, thickness/diameter, and diameter $/ l_{\mathrm{ex}}$. The 


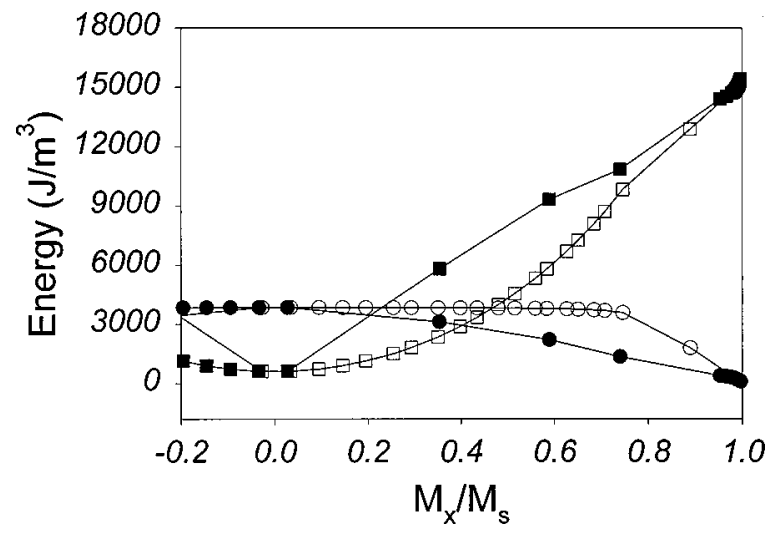

FIG. 3. The demagnetizing energy (square) and the exchange energy (circle) for the $300 \mathrm{~nm}$ diam sample versus the normalized magnetization. The sample thickness is $10 \mathrm{~nm}$. The open symbols are for the increasing field section of the hysteresis loop, while the filled symbols are for the decreasing field section of the loop. The bend in the exchange energy curve at $M_{x} / M_{s} \sim 0.8$ results from vortex annihilation. The corresponding bend in the curve due to vortex formation does not appear in the section of the hysteresis loop shown.

phase diagram for supermalloy given in Fig. 4 shows that there is a distinct line separating the SD and VS "phases." The functional form of this line is a hyperbola. For sample with a diameter $/ l_{\mathrm{ex}}$ ratio below roughly 20 the magnetic domain pattern is single domain regardless of the thickness of the dots, within the range of thickness studied here. Also, as the thickness of the dot decreases the pattern is again single domain for extremely small thickness/diameter ratios regard-

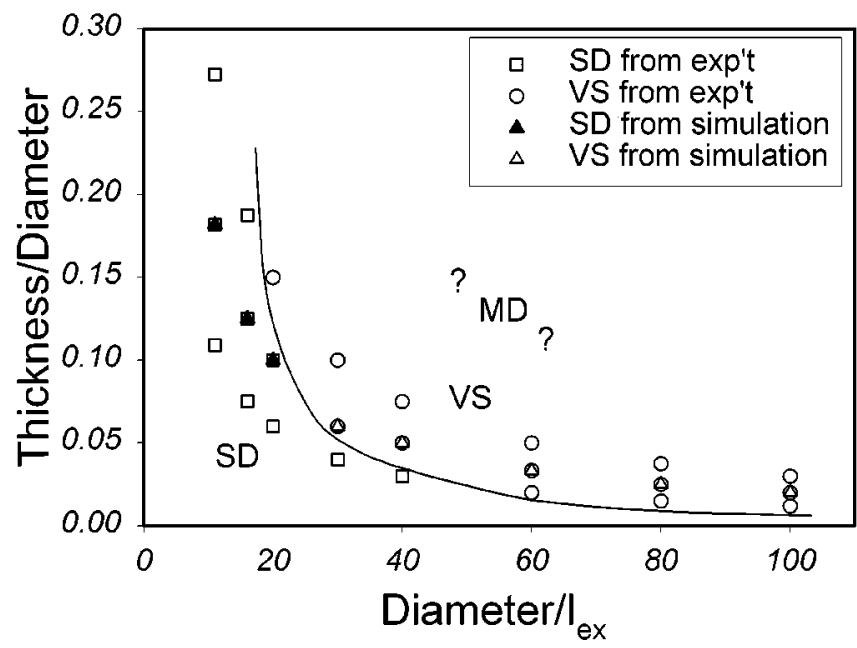

FIG. 4. A phase diagram for supermalloy showing the regions where the sample is in the SD, VS, or MD state as a function of the thickness and diameter of the supermalloy dots. Experimental and simulation results are shown. less of the diameter of the dot. Presumably at sufficiently large diameters and thickness the magnetic domain patterns will be multidomain. This region of the phase diagram was not studied here and is suggested using question marks in Fig. 4. This phase diagram is similar to the previously published diagram for permalloy. ${ }^{4}$

\section{CONCLUSIONS}

Overall, the simulated hysteresis loops are in good agreement with the experimental loops. For all dot diameters supporting vortex formation the nucleation field obtained from the simulation agrees with the experimental value to within 20 Oe. At the largest diameter, $500 \mathrm{~nm}$, the simulated hysteresis loop is exactly the same as the experimental loop, the nucleation field agrees within 2 Oe. The size of the sample is critical. For thin or low diameter dots, the dot cannot sustain a vortex and a single domain pattern with all spins essentially aligned in one direction is obtained. For these dots, the large difference in coercive field is probably due to imperfection in the experimental samples, leading to premature switching. It is also possible that these differences are due to gridding artifacts in the simulations. For large diameter dots or thick samples, the hysteresis loops corresponds to the creation and annihilation of a vortex state. As observed in Fig. 4, the line separating the single domain "phase" from the vortex state phase is hyperbolic. Dots with a diameter-to-exchange-length ratio below roughly 20 will be single domain regardless of the thickness, within the range of thickness measured here.

\section{ACKNOWLEDGMENTS}

The authors wish to acknowledge the support of DARPA via DOD/DARPA MDA972-97-1-0003. The authors also wish to acknowledge the Department of Engineering, University of Cambridge, Cambridge.

${ }^{1}$ J. F. Smyth, S. Schultz, D. Kern, H. Schmid, and D. Yee, J. Appl. Phys. 63, 4237 (1988)

${ }^{2}$ A. Hubert and M. Ruhrig, J. Appl. Phys. 69, 6072 (1991).

${ }^{3}$ P. Vavassori, O. Donzelli, V. Metlushko, M. Grimsditch, B. Ilic, P. Neuzil, and R. Kumar, J. Appl. Phys. 88, 999 (2000).

${ }^{4}$ C. Miramond, C. Fermon, F. Rousseaux, D. Decanini, and F. Carcenac, J. Magn. Magn. Mater. 165, 500 (1997).

${ }^{5}$ R. P. Cowburn, D. K. Koltsov, A. O. Adeyeye, M. E. Welland, and D. M. Tricker, Phys. Rev. Lett. 83, 1042 (1999).

${ }^{6}$ T. Pokhil, D. Song, and J. Nowak, J. Appl. Phys. 87, 6319 (2000).

${ }^{7}$ http://math.nist.gov/oommf

${ }^{8}$ N. Dao, S. R. Homer, and S. L. Whittenburg, J. Appl. Phys. 86, 3262 (1999); C. T. Yu, H. Jiang, P. J. Flanders, and G. J. Mankey, ibid. 87, 6322 (2000); J. Raabe, R. Pulwey, R. Sattler, T. Schweinbock, J. Zweck, and D. Weiss, ibid. 88, 4437 (2000).

${ }^{9}$ M. J. Donahue and R. D. McMichael, Physica B 233, 272 (1997).

${ }^{10}$ D. V. Berkov, K. Ramstock, and A. Hubert, Phys. Status Solidi A 137, 207 (1993)

${ }^{11}$ Concise Encyclopedia of Magnetic and Superconducting Materials, edited by J. E. Evetts (Elsevier Science, New York, 1992), p. 356. 